Research Article

\title{
Experimental Estimation of the Elastic Modulus of Concrete Girders from Drive-By Inspections with Force-Balance Accelerometers
}

\author{
Angelo Aloisio $(1)$ and Rocco Alaggio \\ Università degli Studi dell'Aquila, via Giovanni Gronchi 18, 67100 L'Aquila, Italy \\ Correspondence should be addressed to Angelo Aloisio; angelo.aloisio1@univaq.it
}

Received 31 May 2021; Revised 23 June 2021; Accepted 28 July 2021; Published 5 August 2021

Academic Editor: Mohamed A. A. Abdelkareem

Copyright (c) 2021 Angelo Aloisio and Rocco Alaggio. This is an open access article distributed under the Creative Commons Attribution License, which permits unrestricted use, distribution, and reproduction in any medium, provided the original work is properly cited.

\begin{abstract}
Parametric identification of bridges using instrumented vehicles can be challenging, mainly due to the reduced length of the time series associated with the bridge span under test. This research discusses the practicability of a time-domain identification method based on the use of an instrumented vehicle. The highest cross-correlation between the bridge response from an elementary analytical model and the experimental one, acquired by a moving force-balance accelerometer, yields the unknown model parameter. The effect of vehicle-bridge interaction is removed by proper filtering of the signals. Specifically, the authors estimate the elastic moduli of seven prestressed concrete bridges and compare a subset of the results to the outcomes of static load tests carried out on the same bridges. There is a good correlation between the elastic moduli from the instrumented vehicle and those from static load tests: the method grasps the approximate value of the elastic modulus of concrete. Still, the data do not return an excellent match due to the bias in the estimation of the deflection shape-the paper remarks on the issues faced during the experimental tests and proposes possible enhancements of these procedures.
\end{abstract}

\section{Introduction}

Static load tests are the chief method to assess the bending stiffness of bridges and monitor their integrity. Nevertheless, static load tests, which are mandatory after construction, may be expensive and time-consuming to track the ageing of the infrastructures. The assessments from static load tests possess a reduced level of uncertainty, and their value is indispensable in critical situations.

However, the managing bodies may handle a vast number of infrastructures, and they cannot carry out periodic static load tests on all of them. Therefore, the current methods followed for prioritizing the interventions are derived from routine visual inspections by expert bodies. Despite the indispensability of human expertise, the status of a bridge cannot always stem from visual investigations. Objective, nondestructive tests with low time and money expense should be companions of the current prioritization methods based on visual inspections [1].
The primary outcomes of static load tests are momentdeflection curves, which estimate the bending stiffness and, consequently, the elastic modulus. Besides, in concrete structures, the elastic modulus represents a synthetic parameter, revealing the state of concrete. Nonlinearities in the moment-deflection curve manifest the occurrence of crack openings, possibly due to prestress losses.

The prioritization criteria would benefit from low-cost and rapid methods which can return the same results of static load tests.

The use of a so-called instrumented vehicle would represent a new frontier in the periodic monitoring of infrastructures [2-15]. There are three main approaches in the field of drive-by inspections of bridges. (i) A straightforward method originates from processing the data acquired by a single passage of an instrumented vehicle. (ii) A second method is based on processing multiple passages of an instrumented vehicle on the same deck [16]. (iii) A hybrid approach includes measuring a reference sensor fixed on the 
bridge deck synchronized with the moving one $[17,18]$. Still, most of the research directs at the first approach.

Several scholars identified the fundamental bridge frequency following analytical, numerical, and experimental investigations [19-24]. Yang et al. proved that it is also possible to estimate the bridge frequency with low velocities of the instrumented vehicle [19]. Additionally, fewer researchers successfully extracted the bridge mode shapes from a moving sensor by adopting various signal processing techniques (e.g., short time-frequency domain decomposition or Hilbert transformation) [25-27]. Other researchers have investigated the identification of damping using moving vehicle responses $[13,28]$. Next to the direct natural frequency identification, it is also possible to estimate mechanical parameters of the bridge from drive-by inspections. Explicitly, the single passage of a vehicle equipped with particular sensors may be an indirect method to estimate the bending stiffness, the midspan deflection, the elastic modulus, or any other valuable parameter for the structural safety assessment (e.g., road irregularity [29]). Besides, the adoption of vehicles with different weights and speeds may return the response of the bridge to increasing values of the excitation, as carried out in static load tests.

However, many practical and theoretical limits undermine the complete success of these methods [30].

The direct FFT of the acquired signals provides an estimate of the dominant spectral components. The first and dominant harmonic, which generates the response of a simply supported girder to a moving load, is a low-frequency half-sine. Nevertheless, the frequency of the half-sine, which is, theoretically, the most significant contribution in the signal acquired by the moving sensor, cannot be estimated from peak-peaking. Fourier transform is derived from the orthogonality between harmonic functions. However, orthogonality holds from the integration on the entire period of the harmonic $(-\pi,+\pi)$. Accordingly, FFT cannot return a reliable estimate of a half-sine natural frequency. Therefore, the direct FFT of the signal acquired by the moving sensor does not provide reliable information about the dominant harmonic of the bridge response (see equation (3)). Therefore, the analyst must devise specific procedures to preprocess the signal, by signal duplication, bank filters, and zero paddings, e.g., in order to manifest the contribution of the excitation frequency [31]. Besides, vehicle-bridge interaction phenomena may be beneficial in magnifying the peak associated with the natural frequency of the bridge [32].

In this field, Aloisio et al. [33] developed a parametric identification procedure based on a drive-by inspection with laser sensors. They estimated the bending stiffness of simply supported girders using correlation functions between the recorded time series. The importance of parametric identification lies in the possibility of providing the unknown structural parameters directly, without the need for model updating using the experimental modal parameters.

This paper extends the procedure proposed by Aloisio et al. [33] to drive-by inspections with force-balance accelerometers (FBAs). The originality of this research lies in the elastic modulus identification based on the correlation between the experimental displacement response, obtained by integrating the signal acquired by FBAs, and the simulated one, derived from an elementary analytical model. The authors chose force-balance sensors because they have a low noise level and very high dynamics, and they can measure very low-frequency signals up to the DC component. To the authors' knowledge, most of the scholars used laser sensors $[34,35]$ or piezoelectric accelerometers [36]. Laser sensors do provide the displacement response directly, but the roughness of the road may compromise the quality of the results. Laser measurements require a uniform surface with the same reflectivity properties. Piezoelectric accelerometers are the most used and versatile transducers in the field of structural dynamics, but their bandwidth does not attain the DC component, as in the force-balance accelerometers.

The authors provided a full validation by comparing a subset of the estimated parameters to those obtained from static load tests. The validation of the method fed a discussion about the future perspective of these techniques.

The paper has the following structure: the first section discusses an elementary analytical model used to predict the single-span response; the third section focuses on presenting the case study and the results; the last sections offer a discussion and a few concluding remarks about this research experience.

\section{Methods}

The analytical solution of the equation representative of the dynamic response of a single-span simply supported beam to a concentrated load moving with constant velocity could not be straightforward [37]. However, if the dynamic equation describes the beam response from a reference frame moving with the same velocity of the load, the analytical solution is almost trivial. In this section, the general theory of moving loads introduces the theoretical basis of the procedure as well as the identification method.

2.1. Background. The equation representative of a bridge deck of length $L$, modelled as a single-span Euler-Bernoulli beam subjected to an external force $P(t)$ travelling with constant velocity $c$, can be written as

$$
\rho A \frac{\partial^{2} y(x, t)}{\partial t^{2}}+d \frac{\partial y(x, t)}{\partial t}+E I \frac{\partial^{4} y(x, t)}{\partial x^{4}}=P(t) \delta(x-c t)
$$

where $\rho$ is the mass density, $A$ is the cross-sectional area, $d$ is the damping of the beam, $E$ is Young's modulus, $I$ is the moment of inertia of the beam cross section, $y(x, t)$ is the transverse displacement function of the beam, and $\delta(\cdot)$ is the Dirac delta function.

Young's modulus $E$ is assumed to be constant along the whole beam.

By imposing the following relation between the describing variables $x(t)=c t$ and setting $d=0$, equation (1) turns into 


$$
\frac{E I}{c^{4}} \frac{\mathrm{d}^{4} y(x, t)}{\mathrm{d} t^{4}}+\rho A \frac{\mathrm{d}^{2} y(x, t)}{\mathrm{d} t^{2}}=P .
$$

The assumptions in equation (2) restrict the application to instrumented vehicles moving with a constant velocity $(x(t)=c t)$, without the effect of road roughness and VBI phenomena. Aloisio et al. [33] discussed the limits of these assumptions in the considered case study. The solution of equation (2) can be written as

$$
y(t)=c_{1}+c_{2} t+c_{3} \sin (\lambda t)+c_{4} \cos (\lambda t)+\frac{P}{2 \rho A} t^{2},
$$

where $c_{1}-c_{4}$ are integration constants and $\lambda^{4}=\rho A c^{4} / E I$. Partial differential equation (1) turns into an elementary ordinary differential equation. By imposing the boundary conditions of a simply supported beam in equation (4) expressed in terms of the new independent variable, the integration constants are

$$
\begin{aligned}
y(0) & =0 \\
y^{\prime \prime}(0) & =0 \\
y\left(\frac{L}{c}\right) & =0 \\
y^{\prime \prime}\left(\frac{L}{c}\right) & =0 \\
c_{1} & =-\frac{P}{\lambda^{2}(A \rho)}, \\
c_{2} & =f\left(c_{3}, c_{4}\right)=-\frac{c}{L}\left(\frac{P(L / c)^{2}}{2 A \rho}+c_{3} \sin \left(\frac{\lambda L}{c}\right)+c_{4} \cos \left(\frac{\lambda L}{c}\right)+c_{1}\right), \\
c_{3} & =\frac{1}{\lambda^{2} \sin (\lambda L / c)}\left[\frac{P}{A \rho}-\frac{P \cos (\lambda L / c)}{A \rho}\right], \\
c_{4} & =\frac{P}{\lambda^{2}(A \rho)} .
\end{aligned}
$$

Equation (2) does not simulate VBI phenomena, but proper preprocessing of the acquired signal can reduce these effects and ease the identification problem. The next section presents a preliminary discussion about the above solution on the considered case study, by isolating the contribution of each of the four terms to the structural response.

2.2. Identification Method. The identification of the unknown modelling parameters is obtained from the correlation between the simulated displacement response and the experimental one obtained from the double integration of the acceleration time-history. The rank correlation [38] between the solution of equation (2) and the experimental measurements can be expressed as

$$
C(\mathbf{X})=\operatorname{corr}\left(\mathbf{y}_{s}, \mathbf{y}_{e}\right),
$$

where $\mathbf{X}$ collects the optimization variables, while $\mathbf{y}_{s}$ and $\mathbf{y}_{e}$ are the discretized displacement responses of the simulated and experimental deflection, respectively. The rank correlation coefficient measures the degree of similarity between $\mathbf{y}_{s}$ and $\mathbf{y}_{e}$, and it is used to assess the significance of the relation between them. The correlation is defined as

$$
\operatorname{corr}\left(\mathbf{y}_{s}, \mathbf{y}_{e}\right)=\frac{\mathbf{y}_{s} \cdot \mathbf{y}_{e}}{\left|\mathbf{y}_{s}\right| \cdot\left|\mathbf{y}_{e}\right|}
$$

where $(\cdot)$ is the inner product and is the norm operator. The parameters which yield the maximum correlation in equation (9) are chosen as optimum parameters:

$$
\widehat{\mathbf{X}}=\underset{\mathbf{X}}{\arg \min } \operatorname{corr}\left(\mathbf{y}_{s}, \mathbf{y}_{e}\right) \text {, }
$$

where $\mathbf{X}$ collects the unknown parameters. In this paper, the authors chose the elastic modulus $(E)$ as the unknown parameter.

\section{Introduction to the Case Study}

The paper deals with the dynamic response of simply supported girders of an Italian motorway, the A24, which connects Rome to Teramo. The motorway, also known as Park Motorway, crosses the Apennine range and has several viaducts. After the Gran Sasso tunnel, the highway is almost a sequence of viaducts made of simply supported prestressed concrete (PSC) girders. The girders, built between the 80s and the 90s, have a trapezoidal-like hollow cross section, as depicted in Figure 1(a). The cross section, almost $2 \mathrm{~m}$ high, is $11.8 \mathrm{~m}$ wide with two large cantilevered wings. The total 


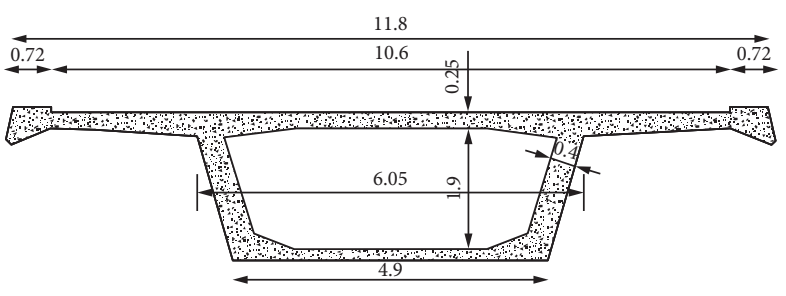

(a)

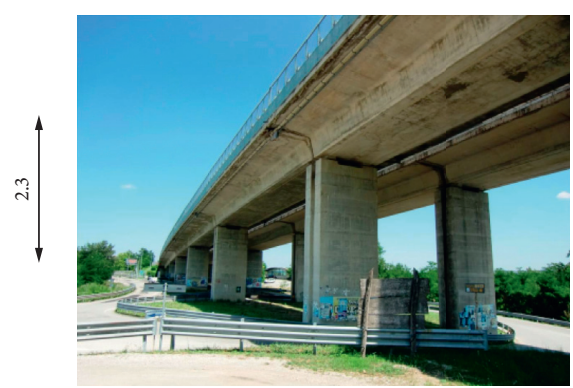

(b)

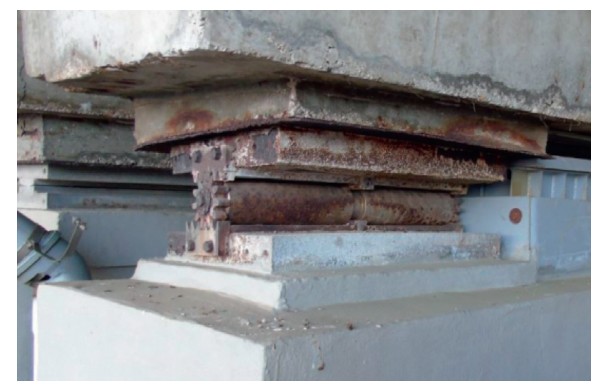

(c)

Figure 1: (a) Cross section of the bridge. (b) View of the Cretara viaduct of the A24 motorway. (c) View of the rack and roller bearing.

length of the beams is $40 \mathrm{~m}$, and they are supported by rack and roller bearings (Figures $1(\mathrm{~b})$ and $1(\mathrm{c})$ ).

The girders were post-tensioned by internal tendons. The designer adopted a limited prestressing condition, i.e., tensile stress under service loads is accepted below the concrete tensile resistance. An approximately constant prestressing force was achieved by symmetrically arranging the tendons to avoid the variation of prestressing due to friction [39]. The prestressing force $T d$ expected at service after the short and long-term losses is about $29000 \mathrm{kN}$. The design modulus of elasticity $E d$ is $35000 \mathrm{MPa}$, while the inertia is approximately $4.338 \mathrm{~m}^{4}$. The prestressing force was not modelled in the analysis, since internal prestressing due to internal bonded tendons has no measurable effects upon the beam dynamics $[40,41]$. The authors focused on the investigation of seven viaducts, also experimented via static load tests.

3.1. Remarks on the Experimental Dynamic Response of the Tested Girders Using Fixed Sensors. The method delivered by this paper is validated using the outcomes of static load tests carried out on seven PSC girders. Additionally, the authors did carry out dynamic measurements of the same seven girders under operational conditions. These paragraphs remark on the most significant outcomes of dynamic identification by discussing the role of bending stiffness in the beams' dynamics. Aloisio et al. $[1,42,43]$ focused on the dynamic identification of a set of seven PSC girders of the A24 motorway. The dynamic identification using two arrays of five accelerometers led to the identification of three stable modes. The first mode resembles the first of a simply supported beam. The second and the third engage the torsional response, as discussed in [42]. Table 1 lists the experimental natural frequencies of the seven spans next to the values of the elastic moduli. Further details about the experimental setup, the identification algorithm, and the modal parameters can be found in $[1,42,43]$.

Hereafter follows a few details extracted from the aforementioned research studies, which may support the reader through the paper.

The first mode shape bestows the prominent contribution to the beam response to a moving load, as can be noted from the spectral analysis of the acquired signal in Figure 2.

Therefore, the estimation of the first mode would guarantee a reliable prediction of the structural response. The tested beams were nominally identical, but their first natural frequencies were very scattered. Conversely, the first mode shapes were very alike, yielding a cross MAC (Modal Assurance Criterion) higher than 0.99. The authors concluded that the first mode shapes almost correspond to those of a simply supported beam, while the possibly diverse boundary conditions, due to the different height of the piles or various supporting devices, did not cause measurable effects on the first modes. Interestingly, the differences in the natural frequencies originate from a significant scatter in the elastic moduli. The discrepancy of the EM may originate from the construction time, when different curing and environmental conditions affected the concrete hardening. The ageing of concrete was not uniform, thus compelling the monitoring of the structural response and, specifically, of the elastic modulus.

3.2. Experimental Equipment. The experimental apparatus consists of a vehicle (Jeep Renegade $4 \mathrm{x}$ ) equipped with four force-balance accelerometers, as shown in Figure 3. There are multiple accelerometers for comparison purposes, supported by three-point adjustable bases. The sampling rate 
TABLE 1: Comparison between the elastic moduli obtained from the instrumented vehicle and the static load tests (the table reports details about the experimental natural frequencies of the first three identified modes).

\begin{tabular}{|c|c|c|c|c|c|c|c|}
\hline \multirow{2}{*}{ Viaduct } & \multirow{2}{*}{ Span } & \multicolumn{3}{|c|}{ Elastic modulus (MPa) } & \multicolumn{3}{|c|}{ Natural frequencies $(\mathrm{Hz})$} \\
\hline & & Moving sensor & Static tests & Error (\%) & 1st mode & 2nd mode & 3rd mode \\
\hline Biselli & 12 & 28000 & 24900 & 11.07 & 2.66 & 6.08 & 8.61 \\
\hline Cerchiara & 4 & 26000 & 15000 & 42.31 & 2.97 & 5.67 & 8.15 \\
\hline Cerchiara & 7 & 25000 & 23700 & 5.20 & 2.68 & 6.30 & 8.36 \\
\hline Cretara & 9 & 43000 & 26000 & 39.53 & 3.56 & 6.73 & 8.63 \\
\hline Le Grotte & 5 & 35000 & 36000 & -2.86 & 2.66 & 6.29 & 8.31 \\
\hline San Nicola & 10 & 38000 & 26700 & 29.74 & 2.68 & 6.89 & 8.84 \\
\hline Temperino & 6 & 36000 & 35900 & 0.28 & 2.52 & 5.41 & 7.92 \\
\hline
\end{tabular}

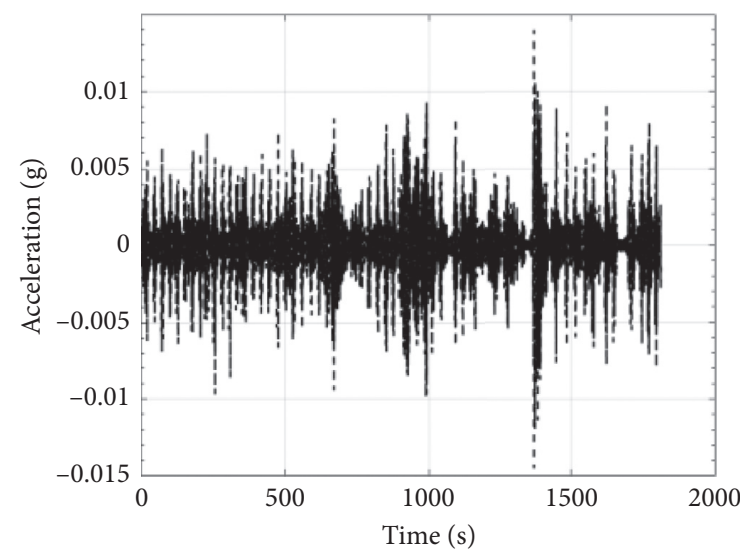

(a)

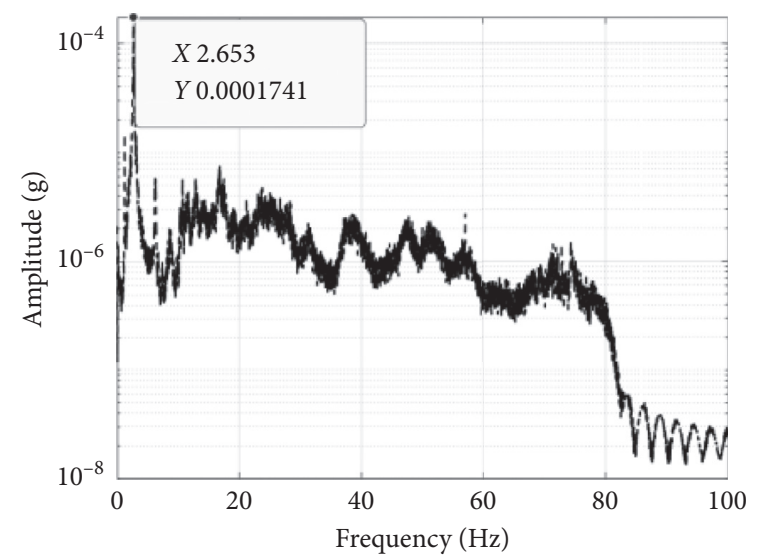

(b)

Figure 2: (a) Acceleration time series acquired by a fixed sensor by the midspan of the Le Grotte viaduct: the peaks correspond to the passage of the vehicle. (b) Fast Fourier transform of the signal in (a).

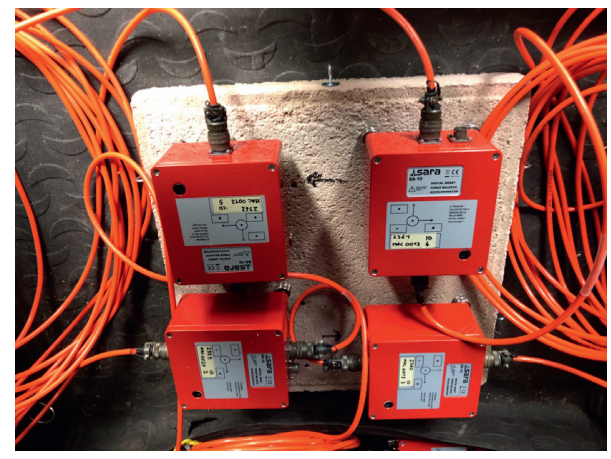

FIgURE 3: Arrangement of the four force-balance accelerometers used in the experimental tests.

is $200 \mathrm{~Hz}$. A lead-acid battery provides power to the accelerometers and the personal computer used for the acquisition. The vehicle's velocity is set constant to $140 \mathrm{~km} / \mathrm{h}$, and it is controlled automatically by embedded cruise control. The signals acquired by the four accelerometers are almost indistinguishable and do not return different outcomes from the identification. Therefore, the results discussed in the last sections descend from a single accelerometer measurement.

Table 2 lists the parameters describing the dynamics of the vehicle and the details of the tested girders needed to simulate the structural response and the preliminary calculations exposed in the following paragraphs.
3.3. Preliminary Calculations. The preceding section presented the exact solution of equation (2) in the case of a simply supported beam. Several scholars faced the moving load equations using the harmonic series. Nevertheless, the projection of the governing equation in the direction $x=c t$ determines the loss of the spatial dimension but returns an elementary ordinary differential equation, which does not require series expansion for the solution estimation. Precisely, the solution of the boundary value problem in the case of a simply supported beam yields the summation of five terms (equation (3)). Four terms are derived from the solution of the homogeneous problem. The fifth is the particular one and originates from the forcing term. The 
TABle 2: Parameters of the instrumented vehicle and the considered bridge stock.

\begin{tabular}{lc}
\hline \multicolumn{2}{c}{ Parameters } \\
\hline Mass of the vehicle & $1750 \mathrm{tn}$ \\
1st natural frequency of the vehicle & $2 \mathrm{~Hz}$ \\
Modal damping of the vehicle & $15 \%$ \\
Velocity of the vehicle & $140 \mathrm{~km} / \mathrm{h}$ \\
Cross section area & $6.6 \mathrm{~m}^{2}$ \\
Bending inertia & $4.4 \mathrm{~m}^{4}$ \\
Length span & $40 \mathrm{~m}$ \\
Mass density & $2500 \mathrm{~kg} / \mathrm{m}^{3}$ \\
\hline
\end{tabular}

manipulation of the analytical expressions in equations (5)-(8) does not deliver straightforward adimensional expressions for a general discussion. Accordingly, the authors present some preliminary calculations, which reveal the contribution of each term in the specific case study using the parameters in Table 2.

Figure 4(a) shows the plot of the girder deflection obtained from the parameters in Table 2. The midspan span deflection is approximately $6 \mathrm{~mm}$ if the elastic modulus is $22000 \mathrm{MPa}$, and the velocity of the vehicle is $140 \mathrm{~km} / \mathrm{h}$. The solution is not a pure half-sine but originates from the summation of five terms. Figure 5 depicts the values of each addend in equation (3), evaluated by the midspan of the girder, whose summation yields the function plotted in Figure 4(a).

Additionally, Figure 5 plots each of the five terms in the entire domain. Variations of the elastic modulus determine notable effects on the midspan deflection, as illustrated in Figure 6. Accordingly, the proposed technique may have adequate premises for a possibly successful estimation.

\section{Results}

The moving accelerometers measured the structural response of 12 viaducts, labelled and itemized in Table 3. Still, the investigation focuses on the analysis of seven viaducts: San Nicola, Le Grotte, Biselli, Cerchiara, Temperino, and Cretara. The seven viaducts are marked in bold in Table 3 . The additional bold phrases, which describe the bridge typology, indicate the specific direction associated with the experimented simply supported girders, namely, L'AquilaTeramo or Teramo-L'Aquila.

The selection of the recordings associated with each viaduct descends from a rough but effective procedure. The experimenter gently hits an accelerometer before and after the passage over the viaduct. The associated spikes allowed the direct and manageable identification of the signals corresponding to each sequence of girders.

Within the selected signal, the identification of the response of each girder is also straightforward. Thermal expansion joints stand between adjacent girders. The joints present a small hump, which causes isolated peaks in the measured response when the wheels cross them.

Figure 7 shows the acceleration signal acquired from the moving sensor in the Le Grotte viaduct. The vertical lines identify the time instants corresponding to the crossing of the thermal joints-the red signal descends from low-pass filtering with a cutoff frequency equal to $0.5 \mathrm{~Hz}$. The effects of the vehicle dynamics manifest at higher frequencies: the first natural frequency of the vehicle is $2 \mathrm{~Hz}$ (see Table 2). The VBI phenomena can be considered negligible, as proved in [33]. Therefore, proper filtering could adequately isolate the bridge response from the vehicle dynamics and other excitation sources (the asphalt roughness), contaminating the dynamics of the girders.

Figure 8 depicts the response of each span of the time series in Figure 7 corresponding to the Le Grotte viaduct. Specifically, Figures $8(\mathrm{a})$ and $8(\mathrm{~b})$ represent the superposition of the acceleration and displacement responses of each span, respectively. The solid red lines indicate the average curves. Figure $8(\mathrm{c})$ shows the simulated bridge response to the moving load along with the displacement responses in Figure 8(b).

The shape of the estimated displacement does not closely agree with the one derived from equation (2). Interestingly, the boundary values of the displacement are approximately zero, which is evidence of the quality of the acquired data and integration. Figure 8(c) has an illustrative purpose. The blue line indicates the theoretical prediction of the displacement response from the direct integration of equation (2) using an elastic modulus equal to $30000 \mathrm{MPa}$. The elastic modulus variation can yield close matching with the maximum displacement value from the experimental curve. The main flaw of this procedure lies in the discrepancy between the shape of the theoretical and simulated response due to the neglection of road roughness and the presence of thermal joints, which causes a jump in the measured acceleration, biasing the integration of the acceleration time-history. The experimental curves in Figure 8 underestimate the displacement by the midspan and overestimate it by the boundary. Likely, the displacement drop by the right boundary originates from the acceleration peak caused by the front wheels crossing the thermal joint, while the rear ones and the accelerometers are still above the girder.

The authors chose not to introduce artificial and possibly arbitrary modifications to the obtained signal to remove this effect. Besides, the length of the vehicle is comparable to that of the girder, and it would not be appropriate to exclude that part of the signal. Likely, the underestimation by the midspan and the overestimation by the right edge may compensate, yielding an accurate estimate. Therefore, the authors attempted to identify the elastic modulus by minimizing the cross-correlation between the simulated and experimental signals. Figure 9 shows the value of the quadratic error between the simulated and experimental signals as a function of the elastic modulus. There are evident minima consistent with the expected value of the elastic modulus of concrete. The legend displays the point of minima associated with each span of the Le Grotte viaduct.

Nevertheless, a few values overestimated the elastic modulus of concrete ( $>6000 \mathrm{MPa}$ ). The authors did not find an exact cause of these biases. Still, several reasons may concur: namely, the nonlinearity of the elastic modulus at higher deformations. Therefore, it is plausible that the elastic modulus of concrete is not independent of the deformation 


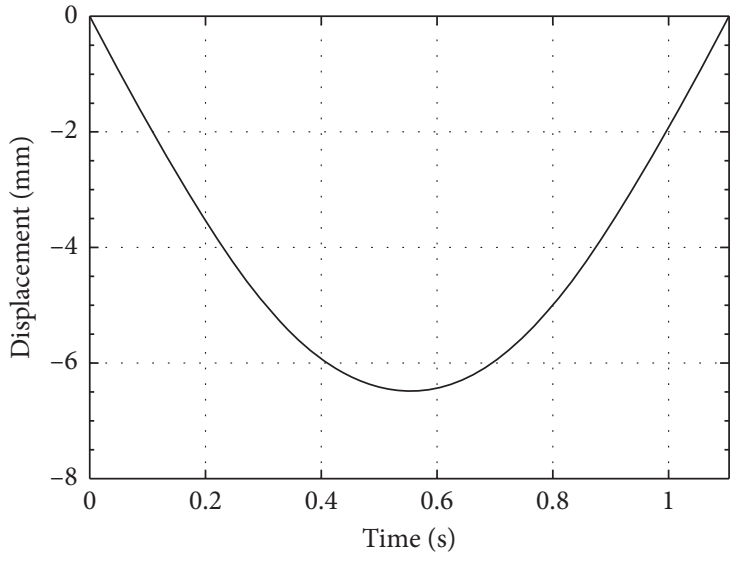

(a)

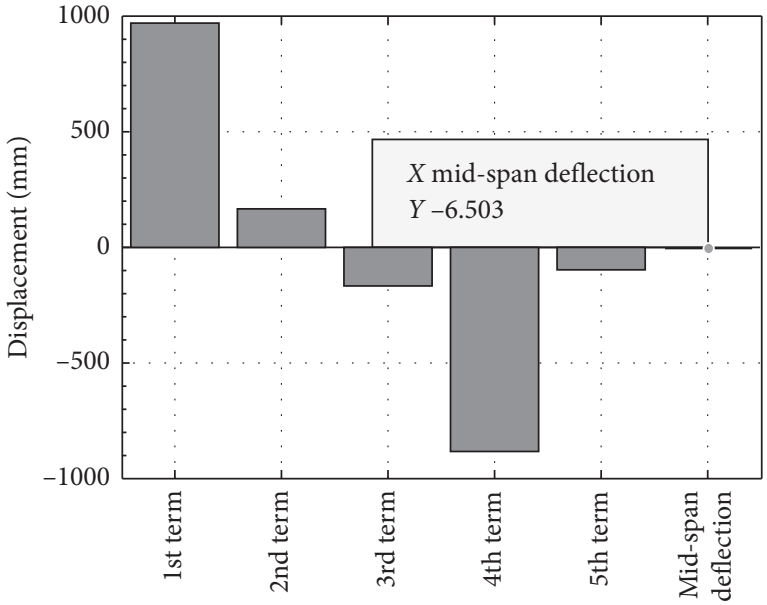

(b)

FIgURE 4: (a) Solution of equation (2) using the parameters in Table 2. (b) Categorical plot of the five terms contributing to the midspan deflection $(\approx 6 \mathrm{~mm})$ with the same order displayed in equation (3).

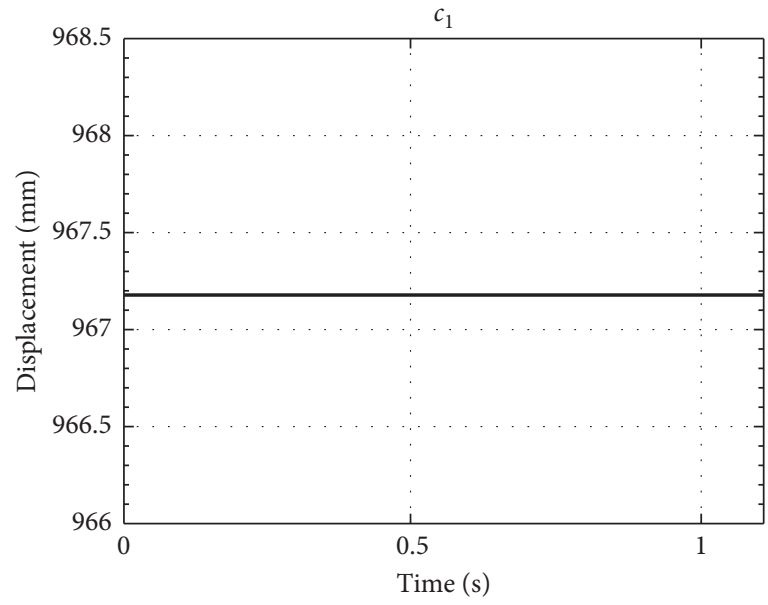

(a)

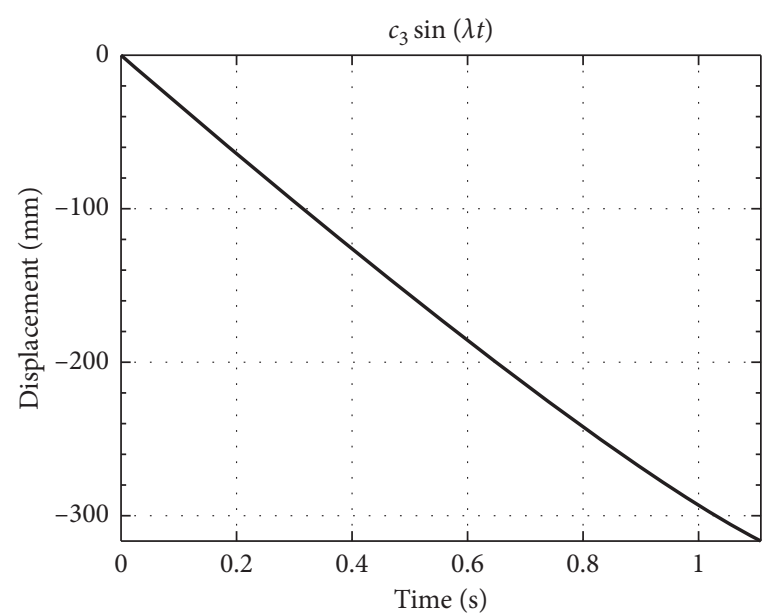

(c)

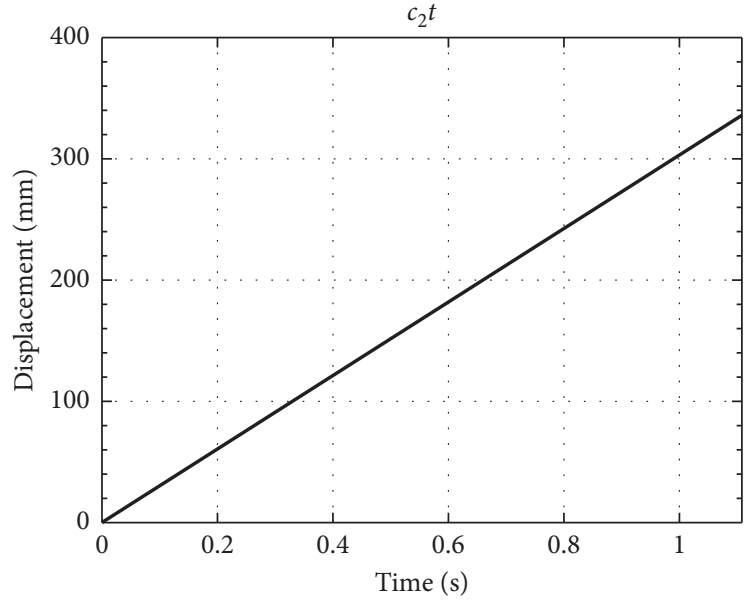

(b)

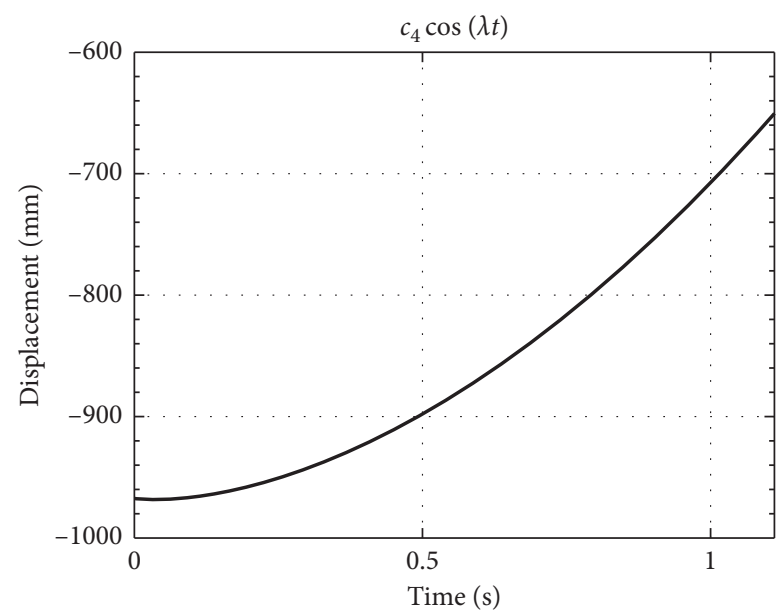

(d)

Figure 5: Continued. 


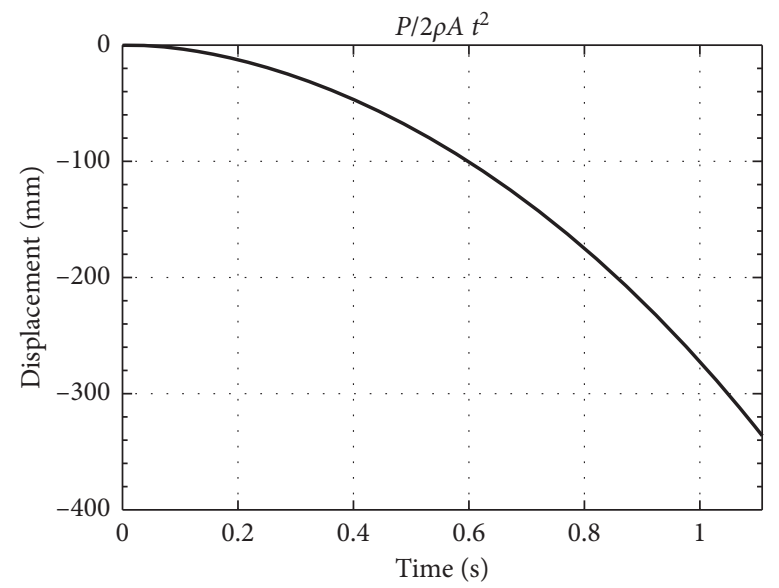

(e)

Figure 5: Plot of the five terms of equation (3) in the considered case study. The title of each subfigure identifies the corresponding term.

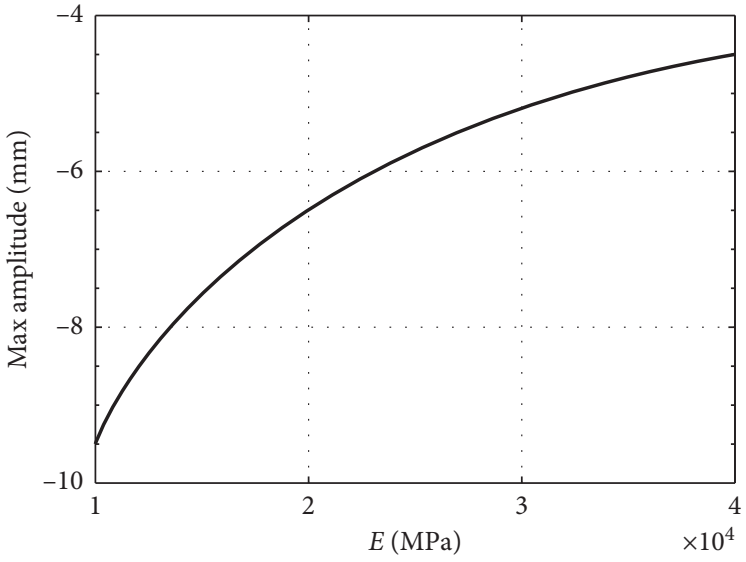

(a)

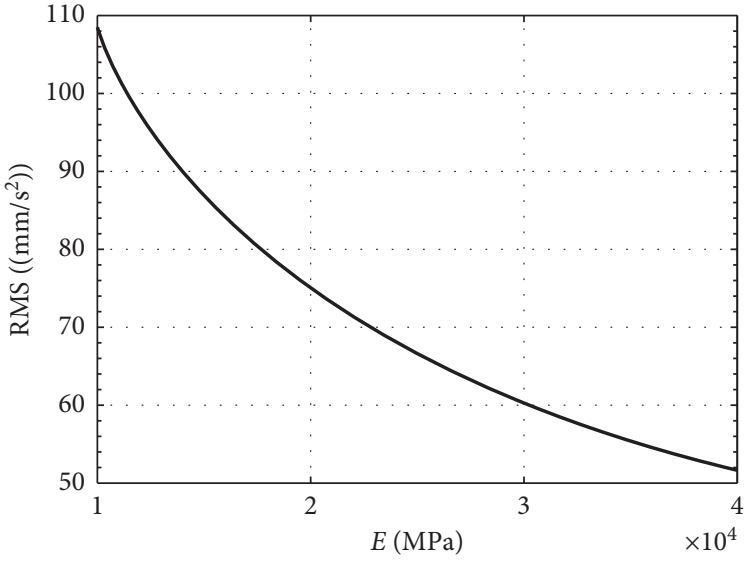

(b)

Figure 6: (a) Midspan deflection of the considered beam element in Table 2, and (b) root mean square (RMS) of the solution of equation (3) as functions of the elastic modulus of concrete.

TABLE 3: Description of the investigated viaducts.

\begin{tabular}{lccc}
\hline Name & No. of spans & L'Aquila-Teramo & Teramo-L'Aquila \\
\hline San Nicola I & 18 & Simply supported girders & Continuous girders \\
San Nicola II & 5 & Simply supported girders & - \\
Valle Situra & 10 & Simply supported girders & Continuous girders \\
Le Grotte & 10 & Simply supported girders & Continuous girders \\
Biselli & 15 & Simply supported girders & Simply supported girders \\
Caldarone & 9 & Simply supported girders & Continuous girders \\
Cerchiara & 29 & Simply supported girders & Simply supported girders \\
Castello & 15 & Continuous girders & Simply supported girders \\
Costa Colle & 13 & Continuous girders & Simply supported girders \\
Temperino & 23 & Continuous girders & Simply supported girders \\
Vico & 6 & Simply supported girders & Simply supported girders \\
Cretara & 12 & Simply supported girders & Simply supported girders \\
\hline
\end{tabular}

The bold character indicates the viaducts tested via static load tests. 


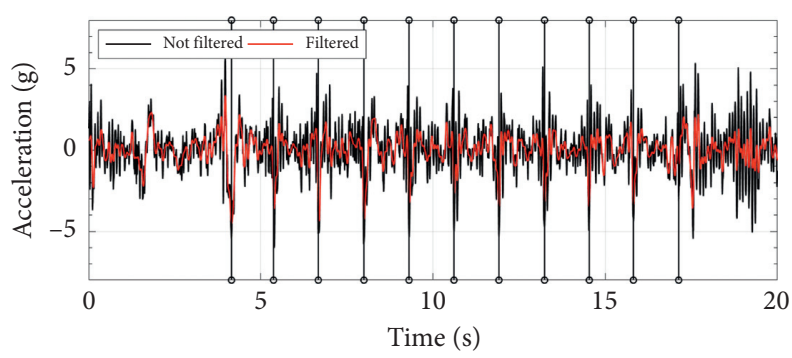

FIgURE 7: Acceleration time series acquired by a moving force-balance accelerometer while crossing the Le Grotte viaduct.

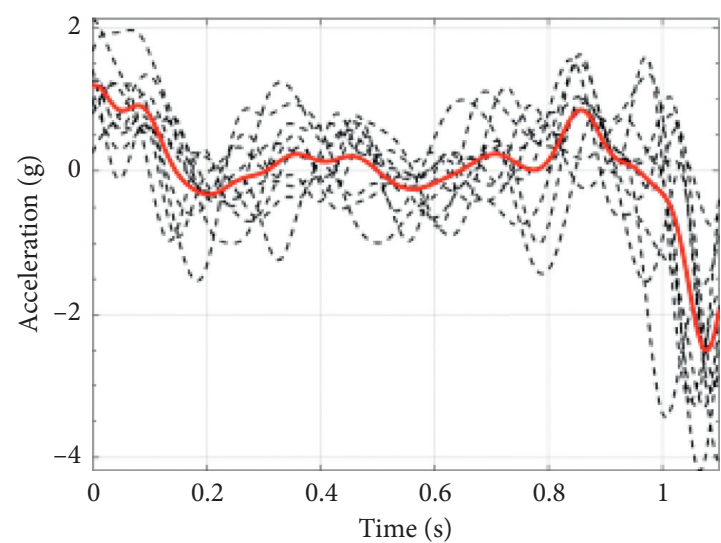

(a)

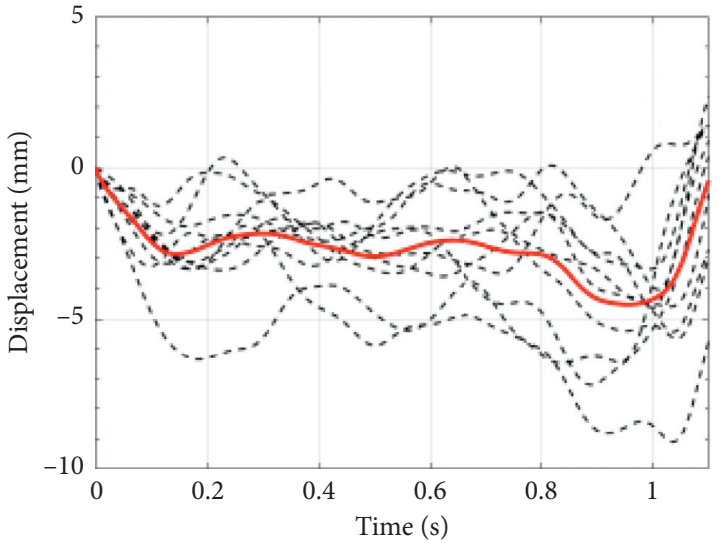

(b)

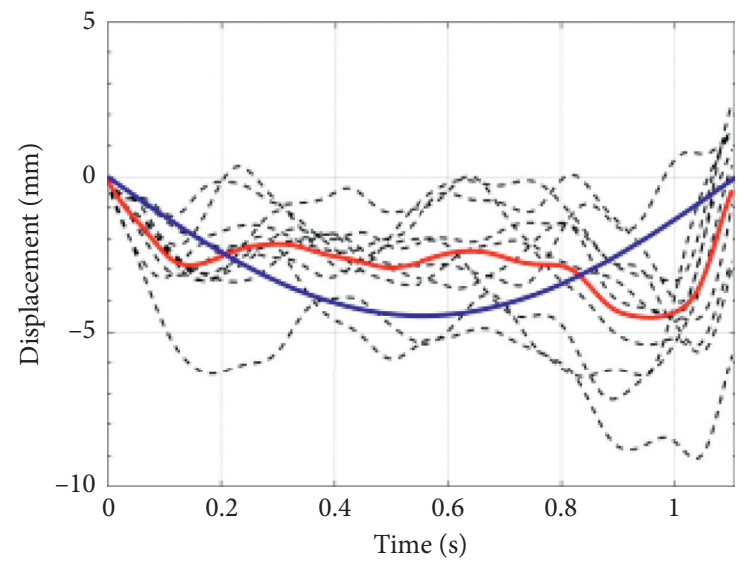

(c)

Figure 8: (a) Superposition of the acceleration time-histories associated with each span of the Le Grotte viaduct (the solid lines represent the average curve). (b) Superposition of the displacement time-histories associated with each span of the Le Grotte viaduct (the solid lines represent the average curve). (c) is the figure in (b) with the blue line representing the optimum theoretical response.

and that lower deformations may be associated with higher values of the elastic modulus, which stabilizes after a certain deformation. The available information is not sufficient to draw definite conclusions. However, the value of span no 5, which is $35000 \mathrm{MPa}$, corresponds to that estimated from the static load test, which is 36000 . This result is encouraging and may endorse the accuracy of the method. However, as shown in Table 1, there are two estimates which do not correspond to the values from static load tests (relative error $\approx 40 \%)$.
As remarked, the nonlinear behaviour of concrete at lower deformation may be the origin of these discrepancies. The authors repeated the measurements to prove the absence of measurement errors. It is likely that some inconsistent values are caused by the irregularities of the road profile. Table 4 exhibits the results from the identification of the chosen girders. The bold values indicate the spans with known elastic modulus from static load tests. Except for a very few cases, the comparison provides a good validation of the method (see Table 1). From a statistical viewpoint, the 


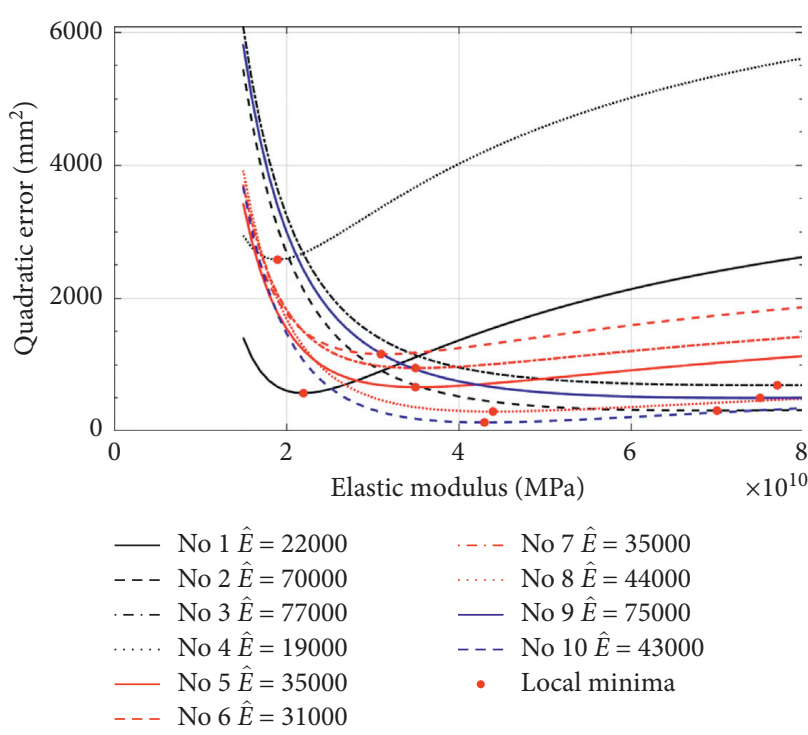

Figure 9: Cross-correlation functions corresponding to the Le Grotte viaduct.

TABLE 4: Elastic moduli in MPa identified using the instrumented vehicle.

\begin{tabular}{|c|c|c|c|c|c|c|}
\hline $\begin{array}{l}\text { Span } \\
\text { no. }\end{array}$ & Biselli & Cerchiara & Cretara & $\begin{array}{c}\text { Le } \\
\text { Grotte }\end{array}$ & $\begin{array}{c}\text { San } \\
\text { Nicola }\end{array}$ & Temperino \\
\hline 1 & 38000 & 18000 & 63000 & 22000 & 40000 & 77000 \\
\hline 2 & 52000 & 43000 & 57000 & 70000 & 52000 & 35000 \\
\hline 3 & 30000 & 27000 & 38000 & 77000 & 29000 & 52000 \\
\hline 4 & 39000 & 26000 & 60000 & 19000 & 39000 & 50000 \\
\hline 5 & 80000 & 30000 & 60000 & 35000 & 50000 & 64000 \\
\hline 6 & 50000 & 34000 & 55000 & 31000 & 21000 & 36000 \\
\hline 7 & 50000 & 25000 & 23000 & 35000 & 42000 & 50000 \\
\hline 8 & 27000 & 36000 & 40000 & 44000 & 35000 & 60000 \\
\hline 9 & 27000 & 80000 & 43000 & 75000 & 38000 & 50000 \\
\hline 10 & 34000 & 43000 & 47000 & 43000 & 57000 & 74000 \\
\hline 11 & 24000 & 31000 & 50000 & - & 51000 & 51000 \\
\hline 12 & 28000 & 56000 & 59000 & - & 51000 & 57000 \\
\hline 13 & 19000 & 50000 & - & - & 41000 & 45000 \\
\hline 14 & 26000 & 27000 & - & - & 18000 & 70000 \\
\hline 15 & 19000 & 39000 & - & - & 50000 & 74000 \\
\hline 16 & - & 63000 & - & - & 37000 & 26000 \\
\hline 17 & - & 27000 & - & - & 39000 & 30000 \\
\hline 18 & - & 47000 & - & - & 28000 & 21000 \\
\hline 19 & - & 77000 & - & - & - & 21000 \\
\hline 20 & - & 80000 & - & - & - & 73000 \\
\hline 21 & - & 68000 & - & - & - & 45000 \\
\hline 22 & - & 42000 & - & - & - & 63000 \\
\hline 23 & - & 27000 & - & - & - & 18000 \\
\hline 24 & - & 42000 & - & - & - & 22000 \\
\hline 25 & - & 24000 & - & - & - & - \\
\hline 26 & - & 35000 & - & - & - & - \\
\hline 27 & - & 18000 & - & - & - & - \\
\hline 28 & - & 22000 & - & - & - & - \\
\hline
\end{tabular}

The bold values correspond to those estimated from static load tests.

method agrees with the expected values significantly. Figure 10 proves that the method leads to a slight overestimation of the elastic modulus. In conclusion, the procedure delivers credible estimates of the elastic modulus, despite a

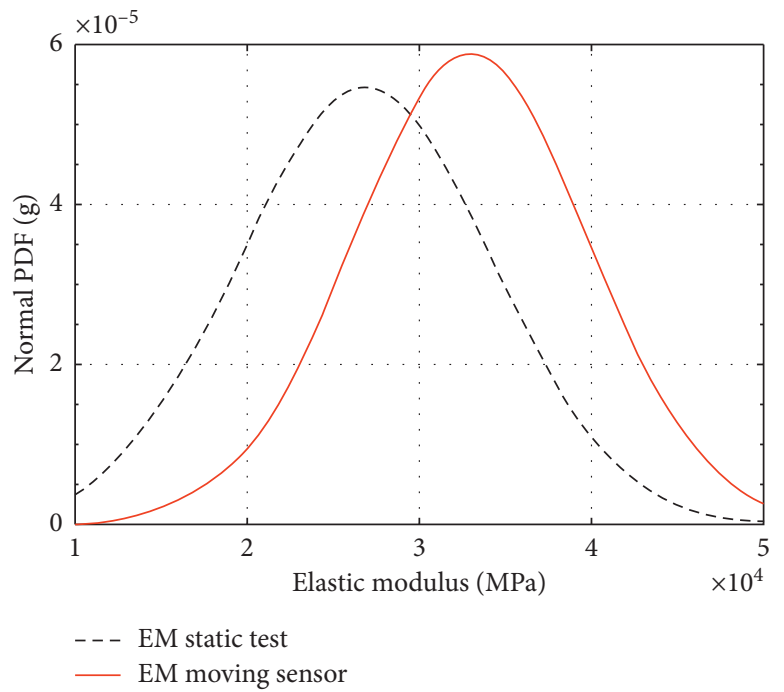

FIGURE 10: Normal probability density function (PDF) of the elastic moduli estimated from static load tests and the moving sensors.

marked shift towards higher values, possibly due to the nonlinearities of concrete at lower deformation, the road irregularities, or other meaningful phenomena neglected in equation (2).

\section{Conclusion}

The paper addresses a notable theme in structural dynamics: parametric identification of bridges from signals acquired by moving sensors. The current research focuses on identifying the elastic modulus of full-scale simply supported prestressed concrete girders using moving force-balance accelerometers. The parametric identification is obtained from the optimization of the cross-correlation between the simulated and experimental displacement responses. The minimum value of the cross-correlation returns the most likely value of the elastic modulus. The authors used an instrumented vehicle, moving with a constant velocity equal to $140 \mathrm{~km} / \mathrm{h}$, equipped with force-balance accelerometers. These accelerometers are characterized by a linear response at very low frequencies up to the DC components. As remarked in the discussion of the governing equations, the estimated signal is almost a half-sine at low frequency. Therefore, force-balance accelerometers may be the most suitable sensors for this purpose. The simulated response is derived from the sole bridge dynamics and does not include vehicle-bridge interaction phenomena, which are negligible in the considered case study [33]. Additionally, the effects of the vehicle dynamics are removed by adopting low-pass filtering with a cutoff frequency lower than the first natural frequency of the vehicle. The outcomes of the static load tests of a subset of the considered spans validated the values obtained from the proposed method. Interestingly, there is a good agreement between the values estimated using the two methods: static load tests and the moving sensor method. In very few cases, the values are inconsistent with the elastic modulus of concrete and present a considerable overestimation. Several phenomena may cause the biases of the 
estimates, and among them, the nonlinear response of concrete even at lower deformation and the road irregularities play a crucial role. Still, despite the presence of a few out-of-range estimates, the method delivers promising results. Future attempts will converge in the estimation of the elastic modulus by varying the velocity and vehicle weight. The estimation of the load curves in bridges, which reports the load as a deflection function, is significant for structural safety assessment and the eventual prioritization of the maintenance interventions. Therefore, the need for agile methods for the indirect estimation of the elastic modulus is a compelling issue, which deserves further research. The current results prove that the proposed procedure still requires further verification for reliable elastic modulus identification.

\section{Data Availability}

The MATLAB code used to support the findings of this study is available from the corresponding author upon reasonable request.

\section{Conflicts of Interest}

The authors declare that they have no conflicts of interest.

\section{References}

[1] A. Aloisio, D. P. Pasca, R. Alaggio, and M. Fragiacomo, "Bayesian estimate of the elastic modulus of concrete box girders from dynamic identification: a statistical framework for the a24 motorway in Italy," Structure and Infrastructure Engineering, vol. 1-13, 2020.

[2] Y. B. Yang and J. P. Yang, "State-of-the-art review on modal identification and damage detection of bridges by moving test vehicles," International Journal of Structural Stability and Dynamics, vol. 18, no. 2, Article ID 1850025, 2018.

[3] M. Makki Alamdari, K. C. Chang, C. W. Kim, K. Kildashti, and H. Kalhori, "Transmissibility performance assessment for drive-by bridge inspection," Engineering Structures, vol. 242, Article ID 112485, 2021.

[4] J. Q. Bu, S. S. Law, and X. Q. Zhu, "Innovative bridge condition assessment from dynamic response of a passing vehicle," Journal of Engineering Mechanics, vol. 132, no. 12, pp. 1372-1379, 2006.

[5] C.-W. Kim and M. Kawatani, "Pseudo-static approach for damage identification of bridges based on coupling vibration with a moving vehicle," Structure and infrastructure engineering, vol. 4, no. 5, pp. 371-379, 2008.

[6] A. González, E. J. O’brien, Y.-Y. Li, and K. Cashell, “The use of vehicle acceleration measurements to estimate road roughness," Vehicle System Dynamics, vol. 46, no. 6, pp. 483-499, 2008.

[7] P. J. McGetrick, A. González, and E. J. OBrien, “Theoretical investigation of the use of a moving vehicle to identify bridge dynamic parameters," Insight - Non-Destructive Testing and Condition Monitoring, vol. 51, no. 8, pp. 433-438, 2009.

[8] H. A. Nasrellah and C. S. Manohar, "A particle filtering approach for structural system identification in vehiclestructure interaction problems," Journal of Sound and Vibration, vol. 329, no. 9, pp. 1289-1309, 2010.
[9] K. V. Nguyen and H. T. Tran, "Multi-cracks detection of a beam-like structure based on the on-vehicle vibration signal and wavelet analysis," Journal of Sound and Vibration, vol. 329, no. 21, pp. 4455-4465, 2010.

[10] S.-H. Yin and C.-Y. Tang, "Identifying cable tension loss and deck damage in a cable-stayed bridge using a moving vehicle," Journal of Vibration and Acoustics, vol. 133, no. 2, 2011.

[11] N. Roveri and A. Carcaterra, "Damage detection in structures under traveling loads by Hilbert-Huang transform," $\mathrm{Me}$ chanical Systems and Signal Processing, vol. 28, pp. 128-144, 2012.

[12] A. Khorram, F. Bakhtiari-Nejad, and M. Rezaeian, "Comparison studies between two wavelet based crack detection methods of a beam subjected to a moving load," International Journal of Engineering Science, vol. 51, pp. 204-215, 2012.

[13] A. González, E. J. OBrien, and P. J. McGetrick, "Identification of damping in a bridge using a moving instrumented vehicle," Journal of Sound and Vibration, vol. 331, no. 18, pp. 41154131, 2012.

[14] L. Majumder and C. Manohar, “"Nonlinear reduced models for beam damage detection using data on moving oscillator-beam interactions," Computers \& Structures, vol. 82, no. 2-3, pp. 301-314, 2004.

[15] S. Sadeghi Eshkevari, S. N. Pakzad, M. Takáč, and T. J. Matarazzo, "Modal identification of bridges using mobile sensors with sparse vibration data," Journal of Engineering Mechanics, vol. 146, no. 4, Article ID 04020011, 2020.

[16] A. Malekjafarian, C. Moloney, and F. Golpayegani, "Drive-by bridge health monitoring using multiple passes and machine learning," European Workshop on Structural Health Monitoring, pp. 695-703, Springer, Berlin, Germany, 2020.

[17] J. Kim and J. P. Lynch, "Experimental analysis of vehiclebridge interaction using a wireless monitoring system and a two-stage system identification technique," Mechanical Systems and Signal Processing, vol. 28, pp. 3-19, 2012.

[18] J. Marulanda, J. M. Caicedo, and P. Thomson, "Modal identification using mobile sensors under ambient excitation," Journal of Computing in Civil Engineering, vol. 31, no. 2, Article ID 04016051, 2017.

[19] Y. B. Yang, H. Xu, B. Zhang, F. Xiong, and Z. L. Wang, "Measuring bridge frequencies by a test vehicle in nonmoving and moving states," Engineering Structures, vol. 203, Article ID 109859, 2020.

[20] C. W. Lin and Y. B. Yang, "Use of a passing vehicle to scan the fundamental bridge frequencies: an experimental verification," Engineering Structures, vol. 27, no. 13, pp. 1865-1878, 2005.

[21] Y.-B. Yang, C. Lin, and J. Yau, "Extracting bridge frequencies from the dynamic response of a passing vehicle," Journal of Sound and Vibration, vol. 272, no. 3-5, pp. 471-493, 2004.

[22] Y. B. Yang and K. C. Chang, "Extracting the bridge frequencies indirectly from a passing vehicle: parametric study," Engineering Structures, vol. 31, no. 10, pp. 2448-2459, 2009a.

[23] K. Kildashti, M. Makki Alamdari, C. W. Kim, W. Gao, and B. Samali, "Drive-by-bridge inspection for damage identification in a cable-stayed bridge: numerical investigations," Engineering Structures, vol. 223, Article ID 110891, 2020.

[24] R. Corbally and A. Malekjafarian, "Examining changes in bridge frequency due to damage using the contact-point response of a passing vehicle," Journal of Structural Integrity and Maintenance, vol. 6, no. 3, pp. 148-158, 2021.

[25] Y. B. Yang, Y. C. Li, and K. C. Chang, "Constructing the mode shapes of a bridge from a passing vehicle: a theoretical study," Smart Structures and Systems, vol. 13, no. 5, pp. 797-819, 2014. 
[26] Y. Yang and K. Chang, "Extraction of bridge frequencies from the dynamic response of a passing vehicle enhanced by the emd technique," Journal of Sound and Vibration, vol. 322, no. 4-5, pp. 718-739, $2009 \mathrm{~b}$.

[27] A. Malekjafarian and E. J. OBrien, "Identification of bridge mode shapes using short time frequency domain decomposition of the responses measured in a passing vehicle," Engineering Structures, vol. 81, pp. 386-397, 2014.

[28] Y. B. Yang, B. Zhang, Y. Chen, Y. Qian, and Y. Wu, "Bridge damping identification by vehicle scanning method," Engineering Structures, vol. 183, pp. 637-645, 2019.

[29] K. Matsuoka, H. Tanaka, K. Kawasaki, C. Somaschini, and A. Collina, "Drive-by methodology to identify resonant bridges using track irregularity measured by high-speed trains," Mechanical Systems and Signal Processing, vol. 158, Article ID 107667, 2021.

[30] D. Hester and A. González, "A discussion on the merits and limitations of using drive-by monitoring to detect localised damage in a bridge," Mechanical Systems and Signal Processing, vol. 90, pp. 234-253, 2017.

[31] Q. Mei, M. Gül, and M. Boay, "Indirect health monitoring of bridges using mel-frequency cepstral coefficients and principal component analysis," Mechanical Systems and Signal Processing, vol. 119, pp. 523-546, 2019.

[32] X. Q. Zhu and S. S. Law, "Structural health monitoring based on vehicle-bridge interaction: accomplishments and challenges," Advances in Structural Engineering, vol. 18, no. 12, pp. 1999-2015, 2015.

[33] A. Aloisio, R. Alaggio, and M. Fragiacomo, "Bending stiffness identification of simply supported girders using an instrumented vehicle: full scale tests, sensitivity analysis, and discussion," Journal of Bridge Engineering, vol. 26, no. 1, Article ID 04020115, 2021b.

[34] S. Artese, V. Achilli, and R. Zinno, "Monitoring of bridges by a laser pointer: dynamic measurement of support rotations and elastic line displacements: methodology and first test," Sensors, vol. 18, no. 2, p. 338, 2018.

[35] A. Malekjafarian, D. Martinez, and E. J. OBrien, "The feasibility of using laser Doppler vibrometer measurements from a passing vehicle for bridge damage detection," Shock and Vibration, vol. 2018, Article ID 9385171, 10 pages, 2018.

[36] A. Malekjafarian, P. J. McGetrick, and E. J. OBrien, “A review of indirect bridge monitoring using passing vehicles," Shock and Vibration, vol. 2015, Article ID 286139, 16 pages, 2015.

[37] L. Frỳba, Vibration of Solids and Structures under Moving Loads, Vol. 1, Springer Science \& Business Media, Berlin, Germany, 2013.

[38] J. Gibbons, Nonparametric Statistical Inference, Marcel Dekker, New York, NY, USA, 2nd edition, 1985.

[39] A. H. Nilson, Design of Prestressed concrete, Vol. 1, Wiley, New York, NY, USA, 1987.

[40] E. Hamed and Y. Frostig, "“Natural frequencies of bonded and unbonded prestressed beams-prestress force effects," Journal of Sound and Vibration, vol. 295, no. 1-2, pp. 28-39, 2006.

[41] A. Aloisio, "Aspects of vibration-based methods for the prestressing estimate in concrete beams with internal bonded or unbonded tendons," Infrastructure, vol. 6, no. 6, p. 83, 2021a.

[42] A. Aloisio, R. Alaggio, and M. Fragiacomo, "Dynamic identification and model updating of full-scale concrete box girders based on the experimental torsional response," Construction and Building Materials, vol. 264, Article ID 120146, 2020a.
[43] A. Aloisio, R. Alaggio, and M. Fragiacomo, "Time-domain identification of the elastic modulus of simply supported box girders under moving loads: method and full-scale validation," Engineering Structures, vol. 215, Article ID 110619, $2020 \mathrm{~b}$. 